\title{
MiR-26b/KPNA2 axis inhibits epithelial ovarian carcinoma proliferation and metastasis through downregulating OCT4
}

\author{
Jiaxin Lin 1,2,*, Lan Zhang 1,2,3,*, He Huang ${ }^{1,2,3, *}$, Yongwen Huang 1,2,3, Long Huang ${ }^{1,2,5}$, \\ Jianhua Wang ${ }^{6}$, Shuting Huang ${ }^{1,2,3}$, Li He ${ }^{1,2,7}$, Yun Zhou ${ }^{1,2,3}$, Weihua Jiaa, ${ }^{1,2}$ Jingping \\ Yun ${ }^{1,2,4}$, Rongzhen Luo ${ }^{1,2,4}$ and Min Zheng ${ }^{1,2,3}$ \\ ${ }^{1}$ State Key Laboratory of Oncology in South China, Guangzhou, P. R. China \\ ${ }^{2}$ Collaborative Innovation Center for Cancer Medicine, Guangzhou, P. R. China \\ 3 Department of Gynecology, Guangzhou, P. R. China \\ ${ }^{4}$ Department of Pathology, Sun Yat-Sen University Cancer Center, Guangzhou, P. R. China \\ ${ }^{5}$ Department of Oncology, The Second Affiliated Hospital, Nanchang University, Nanchang, P. R. China \\ ${ }^{6}$ Cardiovascular Department, Second People's Hospital of Guangdong Province, Guangzhou, P. R. China \\ ${ }^{7}$ Department of Obstetrics and Gynecology, The First Affiliated Hospital, Sun Yat-Sen University, Guangzhou, P. R. China \\ * These authors have contributed equally to this work
}

Correspondence to: Min Zheng, email: zhengmin@sysucc.org.cn

Keywords: epithelial ovarian cancer, KPNA2, miR-26b, OCT4

Received: February 24, $2015 \quad$ Accepted: May 30, $2015 \quad$ Published: June 08, 2015

This is an open-access article distributed under the terms of the Creative Commons Attribution License, which permits unrestricted use, distribution, and reproduction in any medium, provided the original author and source are credited.

\section{ABSTRACT}

Karyopherin alpha 2 (KPNA2) is a nuclear transport protein upregulated in many cancers. Our previous study has identified KPNA2 overexpression in epithelial ovarian carcinoma (EOC) tissues, which predicts poor prognosis. However, the mechanism of KPNA2 overexpression in EOC remains unclear. This study aimed to examine the role of miRNA in KPNA2 dysregulation. Our results showed that miR-26b was downregulated in EOC samples, and correlated inversely with KPNA2 expression. Low expression of miR-26b was associated with advanced FIGO stage, poor differentiation, higher risk of distant metastasis and recurrence. Downregulation of miR-26b predicted poor disease-free survival and overall survival in EOC patients. KPNA2 was validated as a direct target of miR-26b. Knockdown of KPNA2 or ectopic expression of miR-26b could downregulate OCT4, vimentin and upregulate E-cadherin. Reintroduction of KPNA2 partially abrogated the suppression effect induced by miR-26b. We further verified that miR-26b/KPNA2/OCT4 axis inhibited EOC cell viability, migratory ability and sphere-forming capacity in vitro and in vivo. In conclusion, our results reveal that miR-26b is downregulated in EOC, and directly targets KPNA2. miR-26b/ KPNA2 axis suppresses tumor proliferation and metastasis through decreasing OCT4 expression, which is indicative of the important role of miR-26b/KPNA2/OCT4 axis in EOC carcinogenesis and progression.

\section{INTRODUCTION}

Epithelial ovarian carcinoma (EOC) is the most common histological type of ovarian cancer, representing the most fatal gynecological malignancy among women worldwide. It was estimated that 21,980 new cases and 14,270 deaths occured in 2014 as a consequence of EOC [1]. The main reason for the high mortality is the lack of diagnostic methods for early stage detection and effective strategies for treatment. Therefore, understanding the molecular pathogenesis and uncovering molecular biomarkers of EOC would facilitate early detection and improve the survival of ovarian cancer patients.

By using cDNA microarrays, we previously found that karyopherin $\alpha 2$ (KPNA2) was overexpressed in EOC tissues compared to paired normal human ovarian surface epithelial (HOSE) tissues. Subsequent studies have shown that the overexpression of KPNA2 was correlated with a 
poor prognosis in EOC [2] and ovarian malignant germ cell tumors [3]. Additionally, we observed that KPNA2 could promote cell proliferation and tumorigenicity by enhancing c-Myc expression and reducing FOXO3a expression in EOC, suggesting that KPNA2 might be a potential therapeutic target in EOC [4].

Karyopherin $\alpha 2$ (KPNA2), a member of the importin $\alpha$ family, is thought to be an important part of nucleocytoplasmic transport [5-7]. Many recent clinical studies have demonstrated that KPNA2 is upregulated in multiple malignancies and associated with an adverse outcome for the affected patients [8-17]. The biological functions of KPNA2 have been examined in some cancer cell lines; for example, overexpression of KPNA2 in a benign breast cell line could increase cell colony formation ability and migration activity in a manner similar to that in malignant cells [18]. Cell migratory ability and viability can also be enhanced by KPNA2 in lung cancer cell lines [13]. Additionally, knockdown of KPNA2 inhibited the proliferation of cells derived from prostate [14], liver cancer [15] and ovarian cancer [4]. KPNA2 could promote carcinogenesis mainly through the translocation of cancer-associated cargo proteins, including proteins with tumor-suppressive or oncogenic properties. Previous evidence suggested that the nuclear localization of OCT4 is mediated by KPNA2 in mouse ES cells during differentiation into neurons [19]. It's also confirmed that OCT4 and KPNA2 have a strong interaction in ES cells [20]. OCT4 and KPNA2 play an important role in nonsmall-cell lung cancer progression: reduction of KPNA2 expression significantly reduces mRNA and nucleoprotein levels of OCT4. Moreover, knockdown of OCT4 or KPNA2 expression inhibits lung cancer cell proliferation [21].

OCT4 (also known as OCT3/4), encoded by the Pou5fl gene, belongs to the POU transcription factor family [22]. OCT4 is pivotal in maintaining self-renewal and pluripotency of embryonic stem (ES) cells [23]. It is well documented that OCT4 is also highly expressed in many cancer cell types [24], and leads to tumorigenicity, tumor metastasis and recurrence after chemoradiotherapy as a cancer stem cells marker [25]. The first report regarding OCT4 in EOC stem cells demonstrated that the tumorigenic clones were isolated from the ascites of a patient with advanced EOC, which displayed heterogeneity in their clonogenic, tumorigenic, and invasive properties and also exhibited stem and progenitorlike characteristics [26]. A recent study has demonstrated that a sub-population of human EOC cells expresses both Lin 28 and OCT4, and this co-expression pattern is associated with increased tumor grade. Additionally, their combined repression results in synergistic inhibition of EOC cell growth and survival [27]. These studies suggest that OCT4 may be one of the defining features of ovarian cancer stem cells regulating cancer initiation and progression.
Many recent findings have indicated that microRNAs (miRNAs) serve significant functions in almost every biological pathway [28-32], and that abnormal miRNAs expression is associated with human cancers [30, 33-35]. miRNAs are a class of endogenous small (18-24 nt) noncoding single-stranded RNAs that regulate gene expression on post-transcriptional level by binding to the 3'-untranslated region (3'UTR) of messenger RNA (mRNA), leading to mRNA degradation or the suppression of protein translation [36]. Some studies have demonstrated the dysregulation and function of miR-26b in diverse types of cancers [3742]. Additionally, existing researches showed that miR$26 \mathrm{~b}$ accelerates neuronal cell differentiation [43] and osteogenic differentiation of unrestricted somatic stem cells [44]. Human embryonic stem cells and metastatic colorectal cancer cells share the common endogenous human miR-26b. Overexpression of miR-26b leads to the inhibition of cell growth in vitro and in vivo [45]. However, the functional role of miR-26b in EOC has never been reported, and expression data for miR-26b in EOC from microarray profiling has yielded contradictory and controversial findings [46-48]. What's more, the role of KPNA2 together with miR-26b in relation to OCT4 remains obscure.

Although KPNA2 plays an important role in cancers, limited information is available regarding factors that control its expression. Therefore, the molecular mechanisms underlying KPNA2 overexpression remain to be further elucidated. In order to explore the mechanism of KPNA2 dysregulation in EOC, in this study we explored whether miRNAs could suppress KPNA2 expression, and sought to identify the biological behaviors that they affect in EOC cells. Here, we reported that miR-26b expression was repressed in EOC samples, and correlated inversely with KPNA2 expression. Downregulation of miR-26b was associated with aggressive and poor prognostic phenotype in EOC patients. KPNA2 was identified as a direct target of miR-26b. Knockdown of KPNA2 or ectopic expression of miR-26b could markedly enhance endogenous E-cadherin levels, while reduce expression of OCT4 and vimentin. Reintroduction of KPNA2 partially abrogated the suppression effect induced by miR-26b. We further verified that miR-26b/KPNA2/OCT4 axis inhibited EOC cell growth, metastasis and sphere formation in vitro and in vivo.

\section{RESULTS}

\section{An inverse correlation between KPNA2 and miR- 26b expression in EOC}

Our previous studies have shown that KPNA2 is overexpressed in epithelial ovarian cancer and correlates 
Table 1: Correlations between miR-26b expression and clinicopathological features of patients with epithelial ovarian cancer.

\section{Characteristics miR-26b expression}

\begin{tabular}{llll}
\cline { 2 - 4 } & High (\%) & Low (\%) & P-value \\
\hline Age, years & & 16 & \\
$>45$ & 12 & 33 & \\
$\leq 45$ & 36 & &
\end{tabular}

FIGO stage

$\begin{array}{llll}\text { I/II } & 25 & 11 & \mathbf{0 . 0 0 3}\end{array}$

III/IV $\quad 23 \quad 38$

\section{Differentiation}

$\begin{array}{llll}\text { Well } & 10 & 5 & \mathbf{0 . 0 2 1}\end{array}$

$\begin{array}{lll}\text { Moderate } & 17 & 12\end{array}$

Poor $\quad 13 \quad 28$

Missing data $8 \quad 4$

Distant metastasis and recurrence

$\begin{array}{llll}\text { Yes } & 31 & 21 & \mathbf{0 . 0 3 2}\end{array}$

$\begin{array}{lll}\text { No } & 17 & 28\end{array}$

Bold $P$-values indicate significance $(P<0.05)$

with a poor prognosis [2]. In this study, we aimed to explore whether miRNAs are associated with KPNA2 expression.

We initially integrated three computational algorithms, including miRanda, PicTar and TargetScan, to search for miRNAs that target the 3'-UTR of KPNA2 mRNA. According to these prediction analyses, the miR26b-binding site mapped correspondingly to the KPNA2 3'-UTR. Thus, miR-26b was of particular interest to us. To determine whether expression of KPNA2 was associated with miR-26b, we examined the levels of KPNA2 and miR-26b in 93 EOC specimens and observed a moderate inverse correlation $(\mathrm{R}=-0.412 ; P<0.05$; Figure $1 \mathrm{~A})$.

Next, miR-26b expression was measured in 97 human EOCs and 12 HOSE tissues by qRT-PCR. We found that miR-26b was significantly underexpressed in EOC compared with HOSE $(P<0.05$; Figure $1 \mathrm{~B})$. To assess the clinical significance of miR-26b levels, patients were separated into the miR-26b 'high-level' $(n=48)$ and 'low-level' $(n=49)$ groups according to the median value of miR-26b expression. As shown in Table 1, low miR-26b levels was correlated with advanced FIGO (the International Federation of Gynecology and Obstetrics) stage $(P=0.003)$, poor differentiation $(P=0.021)$, higher risk of distant metastasis and recurrence $(P=0.032)$. Kaplan-Meier survival analysis showed that 'low-level' miR-26b group was associated with poor disease-free survival and overall survival in EOC patients $(P<0.05$; Figure 1C, 1D). Moreover, differentiation, the FIGO stage and miR-26b expression were associated with overall 
survival, as indicated by univariate analysis. However, multivariate analysis indicated that differentiation and the FIGO stage were independent prognostic factors for EOC patients, independent of miR-26b expression (Table 2).

\section{KPNA2 is a direct target of miR-26b}

We further explored the inverse correlation between KPNA2 and miR-26b expression in eight EOC cell lines (A2780, HO8910, COV504, COV644, OVCAR-3, OVCAR-4, CAOV-3 and SKOV3). In most of the EOC cell lines, both the mRNA and protein levels of KPNA2 were negatively correlated with miR-26b expression (Figure 2A, 2B). COV644 and OVCAR-3, which showed high levels of KPNA2 expression and low levels of miR$26 \mathrm{~b}$ expression, were transfected with miR-26b mimics. As measured by qRT-PCR and western blotting, ectopic overexpression of miR-26b was observed to suppress endogenous KPNA2 expression at both the mRNA and protein levels when compared to cells transfected with scramble miRNA $(P<0.05$; Figure 2C, 2D).

To characterize whether miR-26b suppresses KPNA2 expression by directly targeting the 3'-UTR, a luciferase assay on $293 \mathrm{~T}$ and OVCAR-3 cell lines was performed. The 3'-UTR of KPNA2 mRNA (pGL3KPNA2-WT) and a mutant variant (pGL3-KPNA2MUT) were constructed and cloned into a luciferase reporter vector (pGL3-control; Figure 2E). When pGL3KPNA2-WT was co-transfected with miR-26b, we found a significant reduction in luciferase activity compared to cells transfected with the scramble miRNA $(P<0.05$, Figure $2 \mathrm{~F}$ ). However, no difference in luciferase activity was observed in cells co-transfected with pGL3-KPNA2MUT and miR-26b. Our findings strongly suggest that KPNA2 expression is suppressed by miR-26b through directly binding.
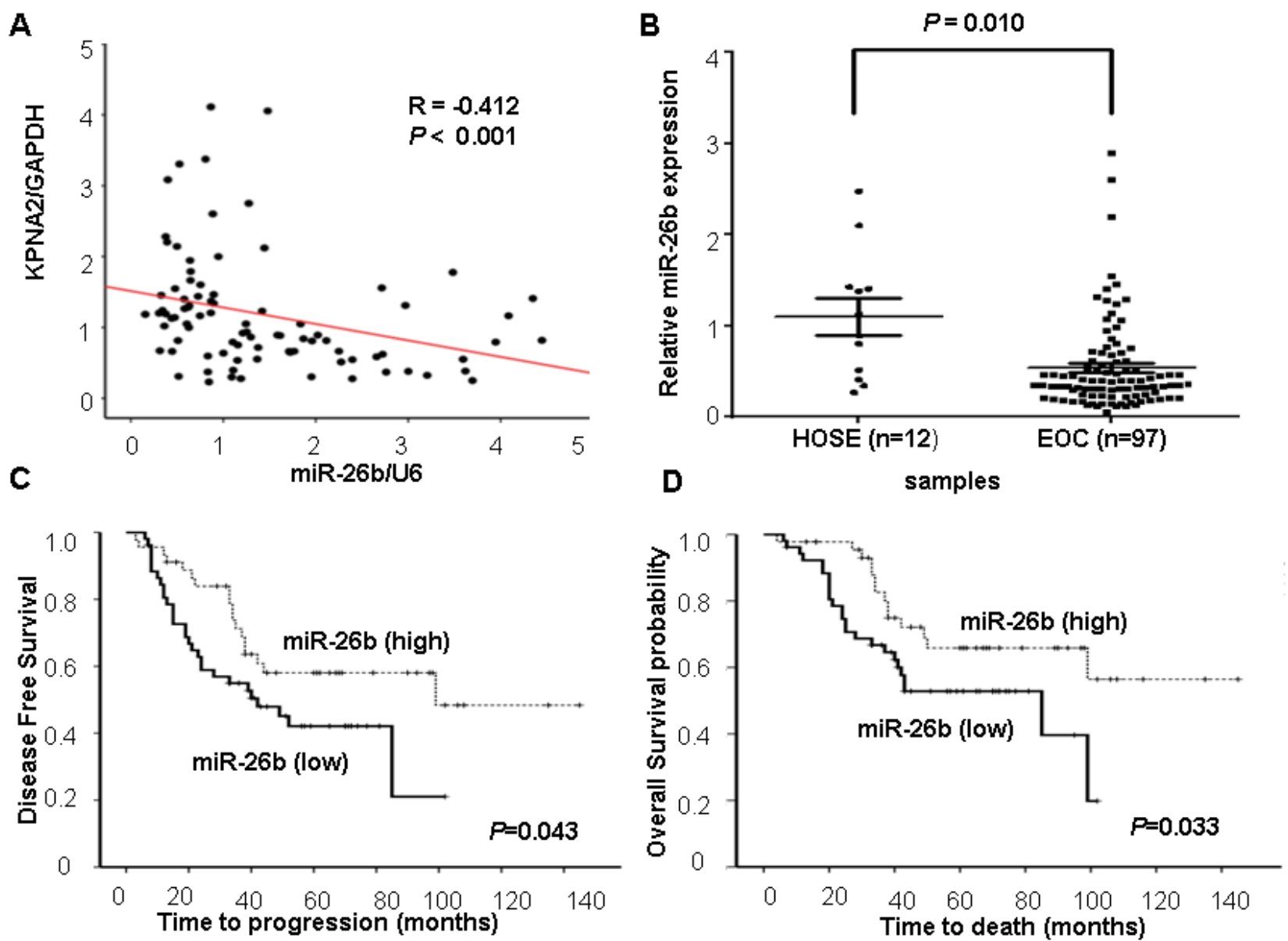

Figure 1: miR-26b expression and its inverse correlation with KPNA2 expression in EOC. A. The correlation between miR$26 \mathrm{~b}$ and KPNA2 expression in EOC tissue samples ( $n=93$; four samples with outlying values were excluded). y, the relative expression of KPNA2 mRNA; GAPDH mRNA was used as an internal control. x, the relative levels of miR-26b; U6 was used as an internal control. Correlations were performed using Pearson's correlation analysis. B. Relative miR-26b expression in 12 HOSE (normal human ovarian surface epithelial tissues) and 97 EOC (epithelial ovarian carcinoma) was determined by qRT-PCR. (C, D) Correlations between miR-26b levels and both disease-free survival and overall survival were tested based on Kaplan-Meier analysis in patients with high $(n=48)$ or low miR-26b expression $(n=49) ; P<0.05$. 
Table 2: Univariate and multivariate analysis of factors associated with overall survival.

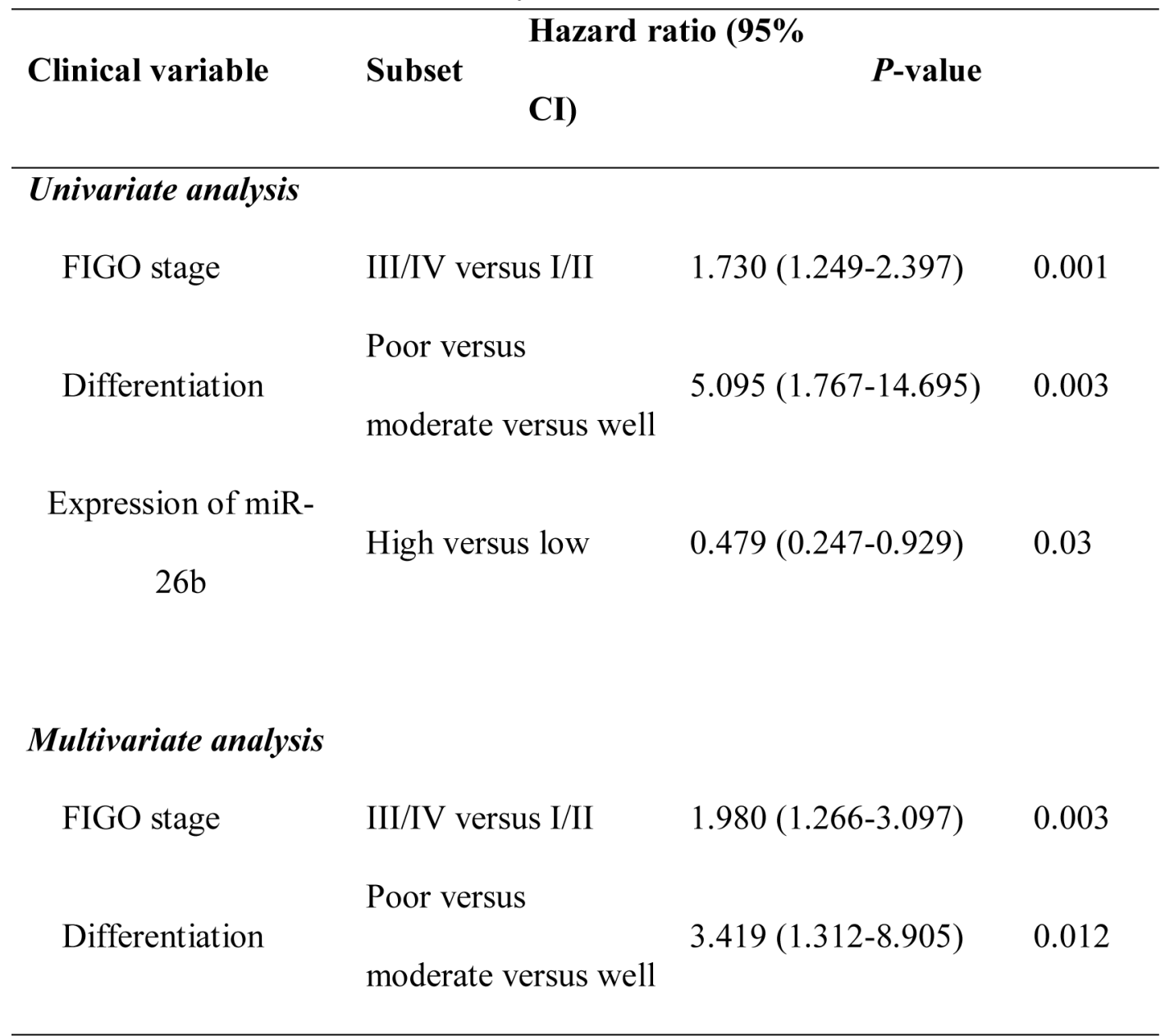

\footnotetext{
Abbreviations: CI, confidence interval; FIGO, International Federation of
}

Gynecology and Obstetrics.

\section{Repression of KPNA2 expression by miR-26b inhibits EOC cell growth, migration and sphere- forming capacity in vitro}

To investigate how miR-26b/KPNA2 axis alters biological behaviors in EOC cells, cell proliferation assay, migration assay and sphere formation assay for the OVCAR-3 cell line were designed. Firstly, overexpression of miR-26b was used to verify that the suppressive effect of miR-26b could mimic that of the reduced expression of KPNA2 (Figure 4A). We found that knockdown of KPNA2 or ectopic expression of miR-26b could significantly inhibit cell viability $(P<0.05$; Figure $3 \mathrm{~A})$, migratory ability $(P<0.05$; Figure $3 \mathrm{~B})$ and sphere-forming capacity $(P<0.05$; Figure 3C) when compared to cells transfected with NC/scramble miRNA.

Subsequently, we tested whether ectopic expression of KPNA2 could rescue the effect of miR-26b. Cells were transfected with KPNA2 siRNA or miR-26b mimics, and followed by pBabe-KPNA2 (encoding the full-length coding sequence of KPNA2 without the 3'-UTR region). KPNA2 levels were again elevated after overexpression of KPNA2 in cells transfected with KPNA2 siRNA or miR-26b mimics (Figure 4A). Reintroduction of KPNA2 partially abrogated the growth arrest, migratory suppression and sphere-forming inhibition induced by miR-26b $(P<0.05$; Figure 3A, 3B, 3C). Western blot analysis showed that si-KPNA2 or miR-26b mimics reduced E-cadherin expression and increased vimentin levels compared with the negative control counterpart (Figure 4A), and the above changes in EMT markers (E-cadherin, vimentin) expression were revoked after KPNA2 reintroduction. This data further proved that miR-26b inhibited EOC cell migration through decreasing KPNA2 expression. Based on our findings therewith, we concluded that the overexpression of KPNA2 caused by the suppression of miR-26b promotes cell proliferation, migration and sphere formation in EOC. 
miR-26b/KPNA2 inhibits EOC cell growth, migration and sphere-forming capacity through decreasing OCT4 expression in vitro

Given OCT4 is translocated by KPNA2 [19-20], we explored whether miR-26b/KPNA2 axis decreases OCT4 expression to inhibit EOC cell growth, migration and sphere-forming capacity. As shown in Figure 4A, OCT4 was downregulated by KPNA2 siRNA or miR-26b mimics. We then tested cell viability, migratory ability and sphereforming capacity in the cells treated with si-OCT4 or siKPNA2. Compared to the negative control, knockdown of KPNA2 or OCT4 expression remarkably reduced the number of viable cells $(P<0.05$; Figure 4B) as well as migratory cells $(P<0.05$; Figure $4 \mathrm{C})$, and formed fewer, smaller spheres $(P<0.05$; Figure 4D). However, the suppression effects by si-OCT4 and si-KPNA2 were abrogated followed by OCT4 reintroduction $(P<0.05$; Figure 4B, 4C, 4D).

Consistently, both si-OCT4 and si-KPNA2 effectively attenuated the protein levels of OCT4 and vimentin, while enhanced E-cadherin expression (Figure $4 \mathrm{E})$. In the cells transfected with si-KPNA2 plus pBabeOCT4, the levels of OCT4 was rescued, with increased vimentin and decreased E-cadherin (Figure 4E).

These data supported that miR-26b/KPNA2/OCT4 axis could inhibit EOC cell proliferation, migration and sphere-forming ability in vitro.
A

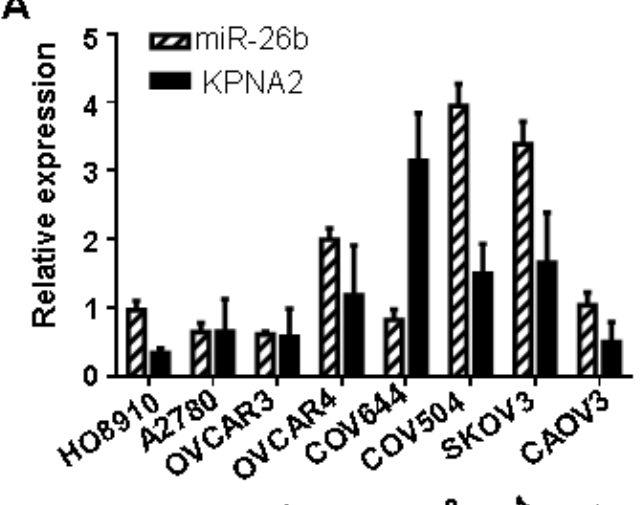

B
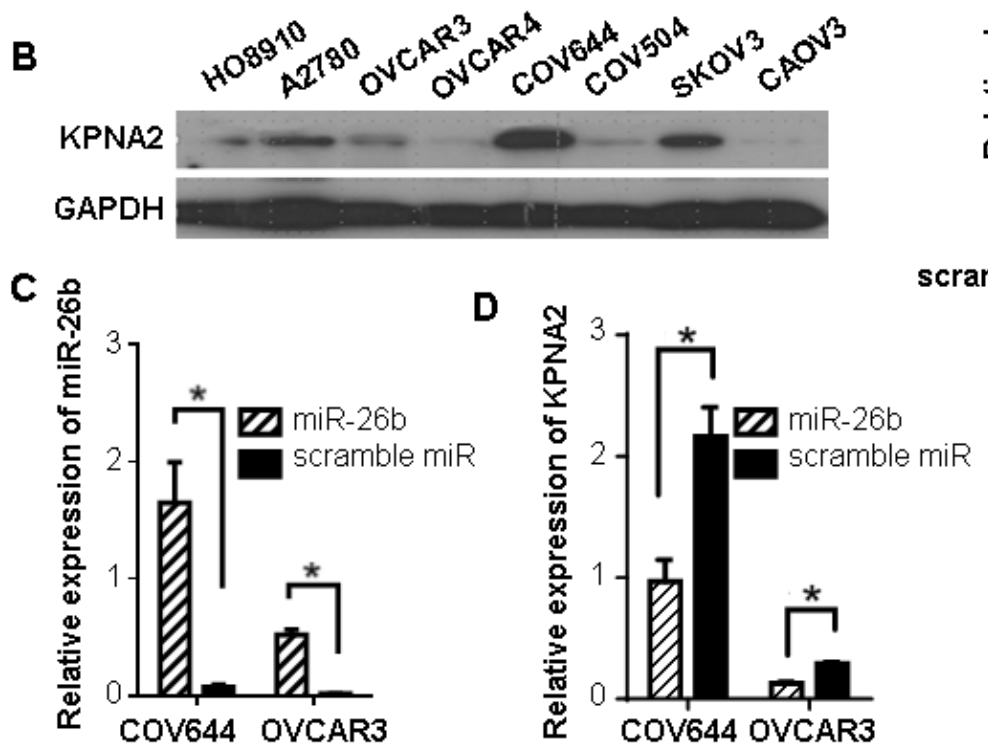

E

\section{$\mathbf{F}$}

pGL3-KPNA2-MUT 5'... UUUACACUGAAACUAGCTGGACC ...3' pGL3- KPNA2-WT 5' ...UUUACACUGAAACUAUACUUGAA ...3' miR-26b 3' UCGGAUAGGACCUAAUGAACUU 5

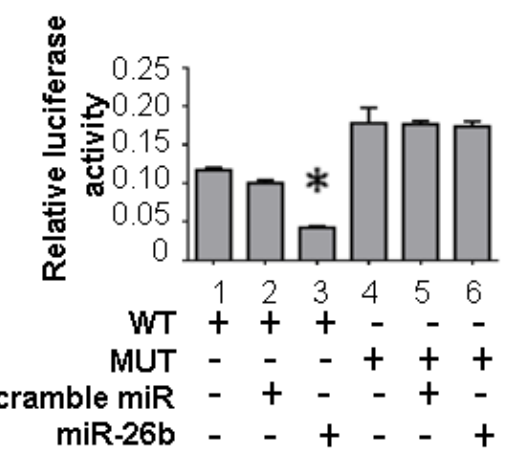

Figure 2: KPNA2 is a direct target of miR-26b. A. The relative $K P N A 2$ mRNA (normalized to GAPDH mRNA), and miR-26b (normalized to U6) expression were detected by qRT-PCR in eight EOC cell lines. B. Relative KPNA2 protein (normalized to GAPDH) levels were measured by western blotting in eight EOC cell lines. C. The levels of miR-26b in COV644 and OVCAR-3 cells significantly increased after transfection with miR-26b mimics, as compared to cells transfected with a scramble miRNA. D. Repression of KPNA2 expression at the post-transcriptional (top) and translational (bottom) levels in COV644 and OVCAR-3 cell lines treated with miR-26b mimics. E. The binding sites of miR-26b in the KPNA2 3'-UTR are shown. The 3'-UTR of KPNA2 mRNA (pGL3-KPNA2-WT) and a mutant variant (pGL3-KPNA2-MUT) were constructed and cloned into a pGL3-control luciferase reporter vector. F. Relative luciferase activity in 293T cells was determined after the WT or MUT 3'-UTR of KPNA2 plasmids were co-transfected with miR-26b mimics or scrambled miRNA. All data are presented as the means \pm SD obtained from three independent experiments; $* P<0.05$. 
miR-26b/KPNA2/OCT4 axis inhibits EOC growth and metastasis in vivo

To determine whether miR-26b was responsible for EOC tumorigenicity, we subcutaneously injected
OVCAR-3 cells stably overexpressing miR-26b or scrambled miRNA into the dorsal flank of nude mice. Compared with control, the tumor volume and weight of the LV-miR26b group were markedly reduced (Figure. 5A, 5B; $P<0.05$ ). As the results of qRT-PCR and $\mathrm{IHC}$ experiments respectively shown in Figure $5 \mathrm{C}$,

A
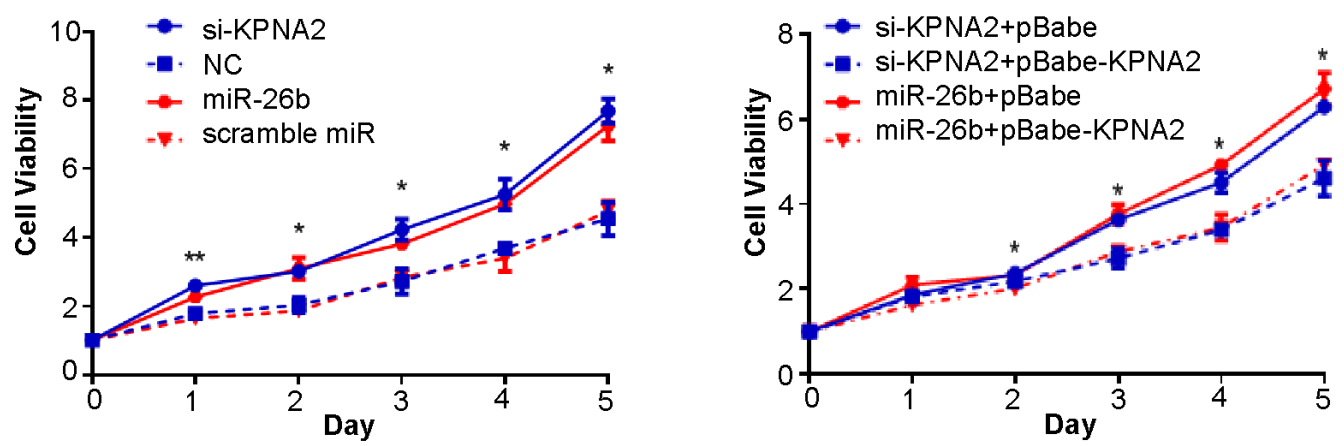

B

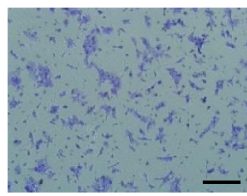

si-KPNA2

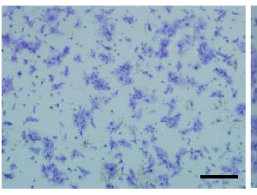

SIKPNA2+

pBabe

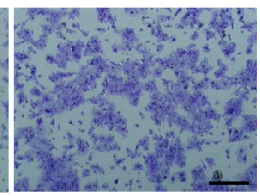

NC

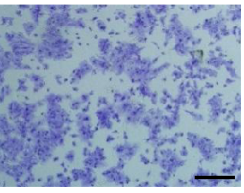

SIKPNA2+ pBabe-KPNA2

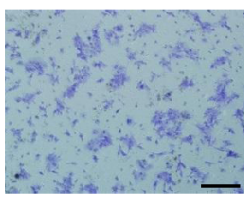

miR-26b

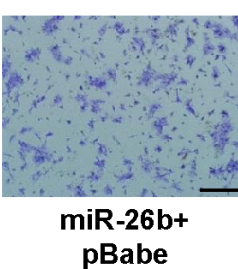

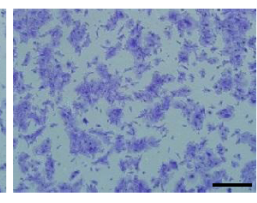

Scramble miR

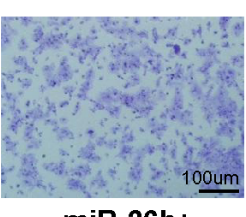

miR-26b+ pBabe-KPNA2
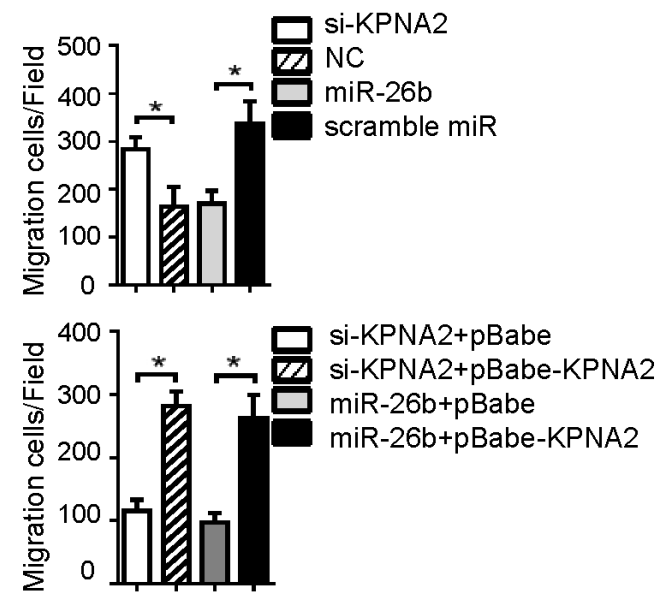

C
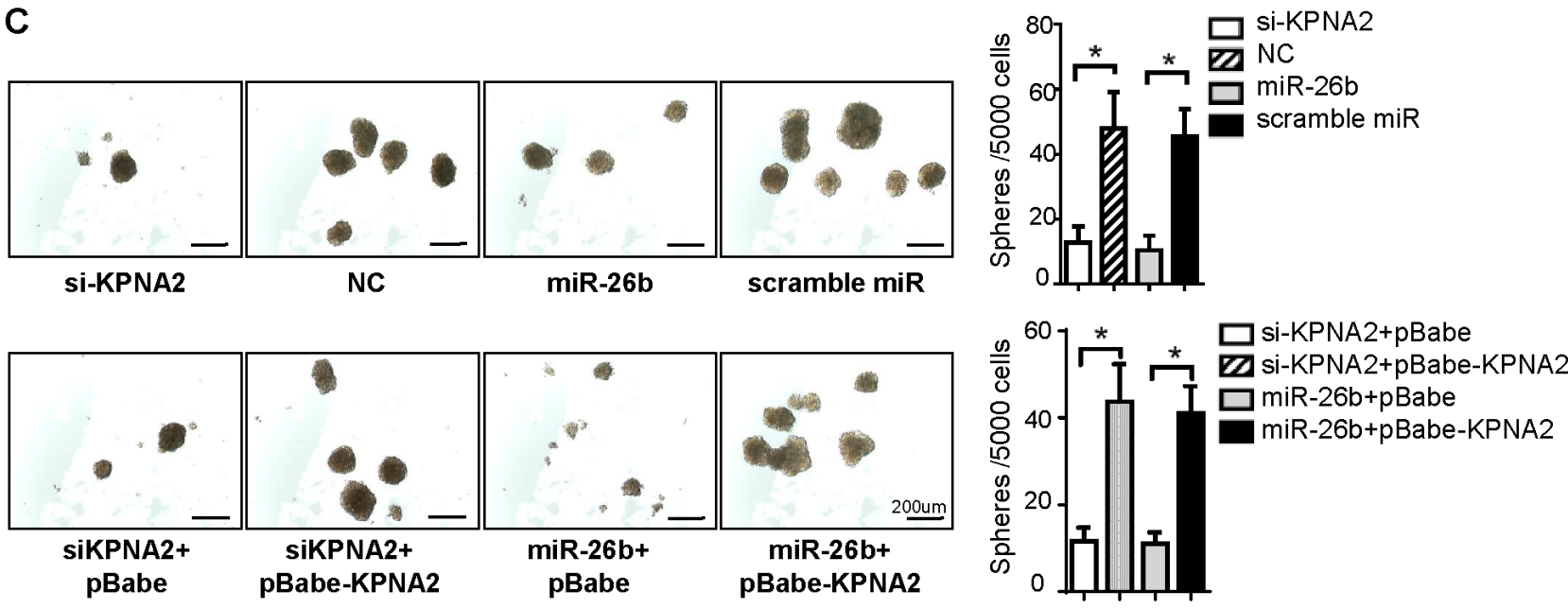

Figure 3: Upregulation of KPNA2 induced by miR-26b reduction promotes EOC cell growth, migration and sphereforming capacity in vitro. A representative MTT assay A., transwell migration assay B. and sphere formation assay $\mathbf{C}$. were performed using the OVCAR-3 cell line. Knockdown of KPNA2 or ectopic expression of miR-26b could significantly inhibit cell viability ( ${ }^{*} P<0.05$ ), migratory ability $(* P<0.05)$ and sphere-forming capacity $\left({ }^{*} P<0.05\right)$ compared to that of cells transfected with NC/scramble miRNA. Reintroduction of KPNA2 partially abrogated the suppression effect induced by miR-26b $\left({ }^{*} P<0.05\right)$. The statistical results of the tumor spheres $(>50 \mu \mathrm{m})$ were calculated. The scale Bars in figure B and C respectively represent $100 \mu \mathrm{m}$ and $200 \mu \mathrm{m}$. All data are representative of three independent experiments. 


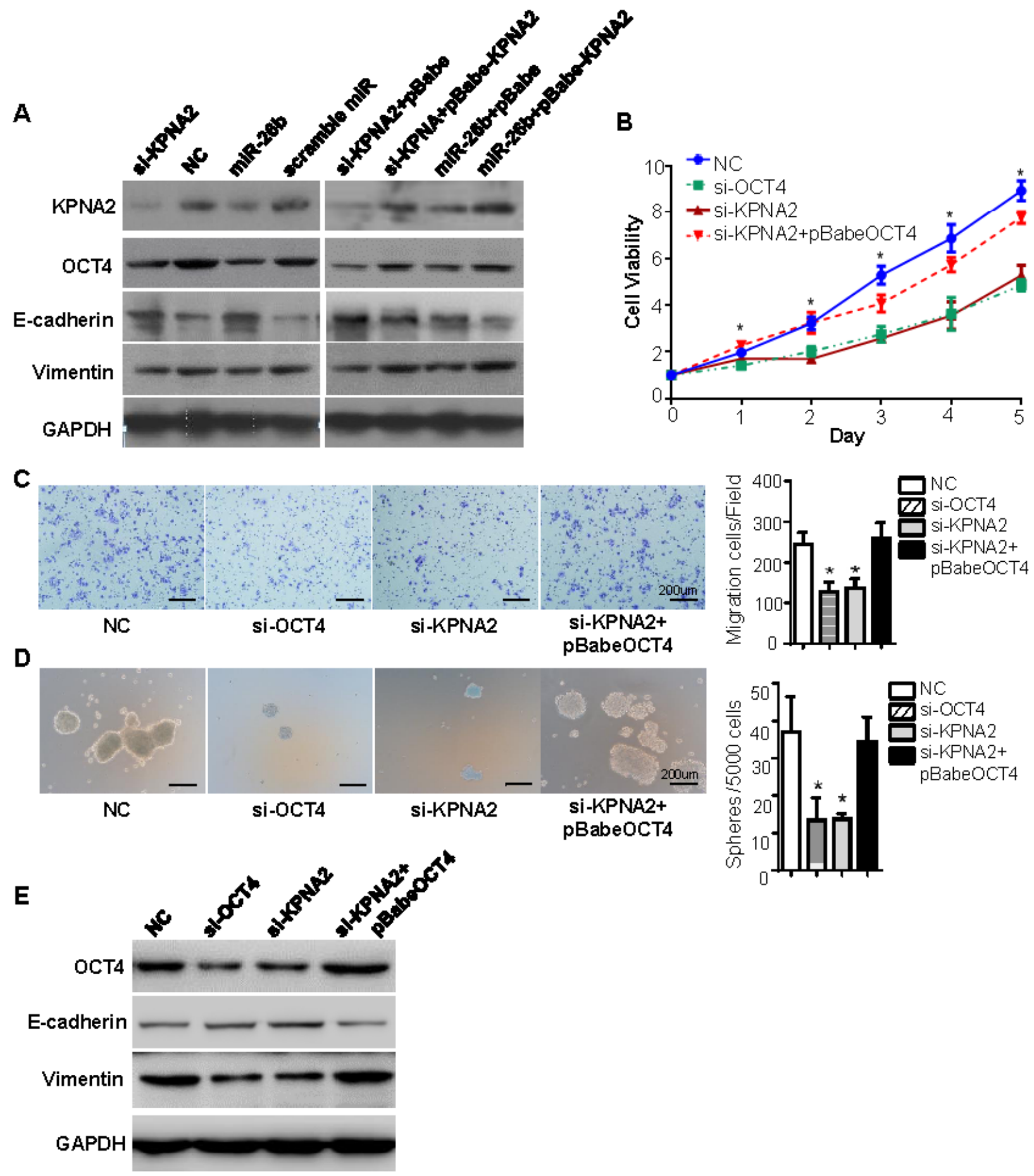

Figure 4: miR-26b/KPNA2 inhibits EOC cell growth, migration and sphere-forming capacity through decreasing OCT4 expression in vitro. A. Western blot analysis revealed that miR-26b mimics could decrease the expression of KPNA2, OCT4, vimentin, and increase E-cadherin levels, similar to the effect of KPNA2 siRNA. The changes of KPNA2, OCT4, vimentin, E-cadherin expression were rescued in OVCAR-3 cells that were transfected with KPNA2 siRNA or miR-26b mimics, followed by pBabe-KPNA2. Knockdown of KPNA2 or OCT4 expression remarkably reduced the viable cells B. as well as migratory cells $\mathbf{C}$., and formed fewer, smaller spheres D.. The suppression effects by si-OCT4 and si-KPNA2 were abrogated followed by OCT4 reintroduction. E. Western blotting was used to detect the protein levels of OCT4, vimentin and E-cadherin in OVCAR-3 cells treated with knockdown of KPNA2 or OCT4 expression, or followed by OCT4 reintroduction. The scale Bars in figure C and D both represent $200 \mu \mathrm{m}$. All experiments were performed in triplicate, and some results are shown as the mean \pm standard deviation. ${ }^{*} P<0.05$. 
5D, the mRNA and protein levels of KPNA2 and OCT4 were significantly lowered in tumors from the miR-26b overexpressing group $(P<0.05)$.

Furthermore, the cells were respectively injected into the tail vein of nude mouse to investigate the effect of miR-26b on metastasis. As shown in Figure 5E, the micrometastases of lung in LV-miR26b group were much fewer and smaller than that of the control group $(P<0.05)$.

In conclusion, miR-26b inhibited EOC growth and metastasis through decreasing the expression of KPNA2 and OCT4 in vivo.

\section{DISCUSSION}

Published reports and our previous studies have established that KPNA2 is highly expressed in multiple malignancies [2, 3, 8-17], and its alterations are often associated with an adverse outcome for breast carcinomas [9-11], esophageal cancer [12], lung cancer [13], prostate cancer [14], hepatocellular carcinoma [15], bladder cancer [16], brain cancer [17] and ovarian cancer [2, 3]. However, the mechanism on how KPNA2 contribute to tumorigenesis and the development of carcinomas remains poorly understood. The biological functions of KPNA2 have been examined in some cancer cell lines; for example, overexpression of KPNA2 in a benign breast cell line could increase cell colony formation ability and migration activity in a manner similar to that in malignant cells [18]. Cell migratory ability and viability can also be enhanced by KPNA2 in lung cancer cell lines [13]. Additionally, knockdown of KPNA2 inhibited the proliferation of cells derived from lung [13], prostate [14], liver cancer [15] and ovarian cancer [4]. Limited data exists on the mechanism of dysregulation of KPNA2. Recently, K1f2 and Klf4 were shown to function redundantly to drive high levels of KPNA2 in mouse embryonic stem cells, which suggested that the regulation of KPNA2 promoter activity is somewhat cell line-specific [49]. Some reports have shown that elevated Kpn $\beta 1$ and Kpn $\alpha 2$ expression in cancer cells correlate with dysregulated E2F/Rb activities [50]. Additionally, E2F1 is translocated in response to KPNA2, raising the possibility of a positive feedback loop. The elevated activity of E2Fs may lead to increased expression of KPNA2, which in turn increases the nuclear abundance of E2Fs [51]. Previously, we found that KPNA2 promotes cell proliferation and tumorigenicity through the upregulation of c-Myc and downregulation of FOXO3a in EOC, suggesting that KPNA2 may be a potential therapeutic target [4].

To explore whether KPNA2 expression in EOC is suppressed by miRNAs, we initially used three computational algorithms to search for miRNAs that target the 3'-UTR of KPNA2 mRNA. We found miR$26 \mathrm{~b}$ level is significantly lower in EOC, and correlated
A

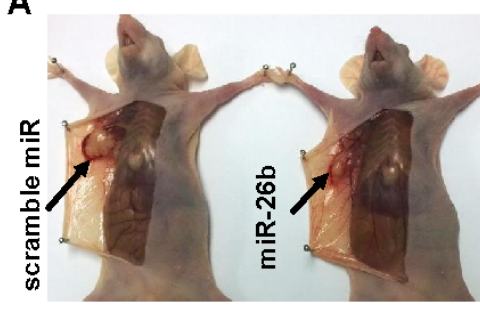

scramble miR miR-26b

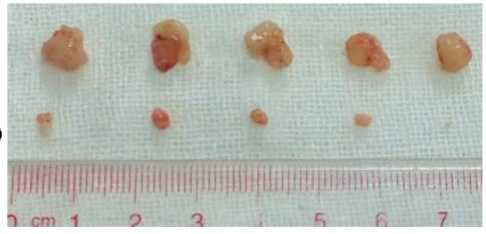

D

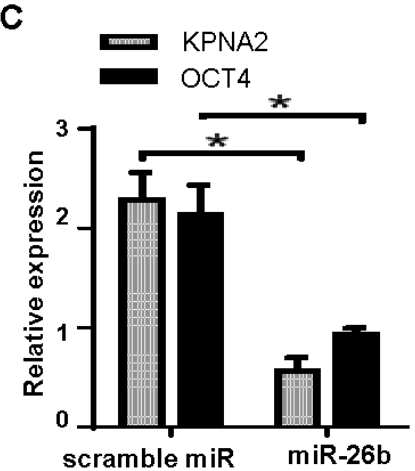

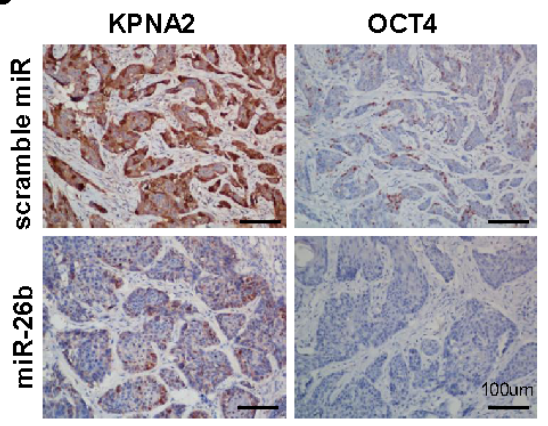

E

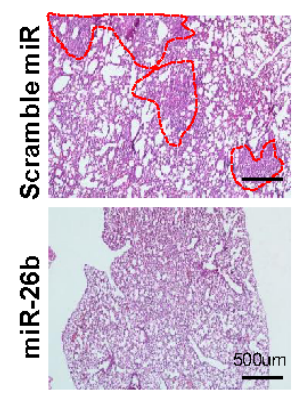

B

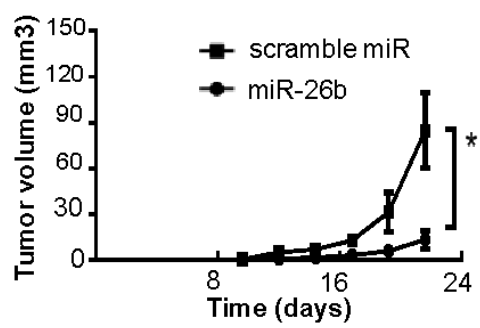

Figure 5: miR-26b/KPNA2/OCT4 axis inhibits EOC growth and metastasis in vivo. A. Representative pictures of xenografts tumor model constructed by subcutaneously injecting OVCAR-3 cells stably overexpressing miR-26b or scrambled miRNA in the dorsal flank of nude mice, and illustration of subcutaneous tumors excised from the mice mentioned above $(n=5)$. B. The tumor growth rate of the experimental mice measured by tumor volumes $\left({ }^{*} P<0.05\right)$. All data are shown as mean \pm S.D. qRT-PCR and IHC were respectively used to detect the mRNA C. and protein D. level of KPNA2 and OCT4 in the mice tumors from miR-26b overexpressing group and control group. The scale bars represent $100 \mu \mathrm{m}$. E. Representative results for H\&E staining of the metastatic nodules in the lung are shown. The metastatic nodules are indicated with dotted line. Bars represent $500 \mu \mathrm{m}$. The statistical result is on the right. $n=6 ;{ }^{*} P<0.05$. 
with FIGO stage, differentiation, distant metastasis, and prognosis of EOC patients. We then demonstrated that there is a moderate inverse correlation between the levels of KPNA2 and miR-26b, which was also confirmed both at the transcriptional and translational levels in the EOC cell lines. When EOC cells were transfected with miR$26 \mathrm{~b}$ mimics, ectopic overexpression of miR-26b markedly suppressed KPNA2 expression at both the mRNA and protein levels when compared to cells transfected with scramble miRNA. We confirmed that KPNA2 is a direct target of miR-26b. Overexpression of KPNA2 caused by the suppression of miR-26b could significantly inhibit cell viability, migratory ability and sphere-forming capacity in vitro. Knockdown of KPNA2 or ectopic expression of miR-26b could downregulate OCT4, vimentin and upregulate E-cadherin. Reintroduction of KPNA2 partially abrogated the suppression effect induced by miR-26b. Additionally, si-OCT4 or si-KPNA2 remarkably reduced the viable cells as well as migratory cells, and formed fewer, smaller spheres. However, the suppression effects were revoked followed by OCT4 reintroduction. This findings support that miR-26b/KPNA2 suppresses EOC cell growth, migration and sphere formation through decreasing OCT4 expression. Furthermore, miR-26b/ KPNA2/OCT4 was confirmed to inhibit EOC proliferation and metastasis in vivo.

Several lines of evidence from literature have provided relevant insight into the dysregulation and function of miR-26b in diverse cancers. In glioma, miR-26b directly reduces EphA2 expression and may act as a tumor suppressor [37]. In human breast cancer, downregulation of miR-26b can suppress cell apoptosis by targeting SLC7A11 [38] and promote cellular growth by binding to PTGS2 [39]. Additionally, miR-26b has also been shown to inhibit colorectal cancer cell growth and invasion, and this effect was partially abrogated by the addition of nicotinamide adenine dinucleotide [40]. Some reports have suggested that miR-26b can suppress $\mathrm{NF}-\kappa \mathrm{B}$ signaling and thereby sensitize hepatocellular carcinoma cells to the doxorubicin-induced apoptosis by inhibiting the expression of TAK1 and TAB3 [41]. Another study reported that miR-26 family members are concomitantly expressed with their host genes (CTDSP1/2/L), and cooperate to block the G1/S-phase transition by synergistically activating the $\mathrm{pRb}$ protein [42]. Contradictory expression data for miR-26b in EOC was reported in some microarray gene expression profiles [46-48]. Our study shows for the first time that miR-26b is significantly underexpressed and exerts its function by directly targeting KPNA2 in EOC.

Some limitations remain within the presented study. First, we were unable to detect obvious reduction in the levels of miR-26b when EOC cell lines were transfected with a miR-26b inhibitor (data not shown). It is possible that the background expression of miR-26b in EOC cells might be too low for such an effect to be observable. Second, the reintroduction of KPNA2 partially abrogated the growth arrest and metastasis suppression induced by miR-26b. As we known, miRNAs can have dynamic interactions with multiple target genes. Hence, other genes might be decreased by miR-26b, as KPNA2 overexpression could not completely counteract the effect of miR-26b. Furthermore, KPNA2 might also be modulated by other miRNAs or regulatory molecules; for instance, long non-coding RNA (lncRNA), a newly identified class of regulatory molecules, is involved in a variety of biological functions. Due to the complexity of control factors in gene expression networks, further investigations are needed to address these issues.

In summary, our studies were the first to identify miR-26b as downregulated and associated with aggressive and poor prognostic phenotype in EOC. KPNA2 is a target gene of miR-26b, and its overexpression in response to miR-26b reduction might promote tumor proliferation and metastasis through increasing OCT4 expression. This link between miR-26b, KPNA2 and OCT4 identifies a novel potential therapeutic target for the treatment of EOC.

\section{MATERIALS AND METHODS}

\section{Human tissue samples and cell lines}

For qRT-PCR experiments, snap-frozen tissues were obtained from 97 EOC patients who underwent surgery at Sun Yat-sen University Cancer Center (Guangzhou, China) between 2002 and 2010. All patients were histologically confirmed and did not receive chemotherapy. All specimens were obtained with the informed consent of patients, and this study was approved by the ethics committee of Sun Yat-sen University Cancer Center.

We obtained eight human EOC cell lines (A2780, HO8910, COV504, COV644, OVCAR-3, OVCAR-4, CAOV-3 and SKOV3) and the human embryonic kidney cell line 293T from the American Type Culture Collection. We cultured the cell lines in Dulbecco's Modified Eagle's medium (DMEM) with 10\% fetal bovine serum (FBS; Invitrogen, Carlsbad, CA, USA).

\section{RNA isolation and quantitative real-time PCR analysis}

Total RNA from tissues and cultured cells was extracted using Trizol (Invitrogen). Reverse transcriptase reactions and qRT-PCR were performed using the M-MLV reverse transcriptase (Promega, Madison, WI, USA) and Platinum SYBR Green qPCR SuperMix-UDG reagents (Invitrogen), respectively. The expression of miR-26b was detected using a Bulge-Loop ${ }^{\mathrm{TM}}$ miRNA qRT-PCR Primer Set (Ribobio, Guangzhou, China) according to the manufacturer's instructions. U6 was used as a 
reference. Primers were designed for KPNA2 (forward, 5'-CTCATAACCATGTCCAC

CAACG-3';

reverse, 5'-CTCTATTCTGCGACGCCTCAT-3') OCT4 (forward, 5'- AGCACGAGTGGAAAGCAACT-3', reverse, 5'-TTCTAGCTCCTTCTGCAGGG-3'), and GAPDH (forward, 5'-CTCCTCCTGTTCGACAGTCAGC-3'; reverse, 5'-CCCA ATACGACCAAATCCGTT-3'). $G A P D H$ was used as an internal control. The relative fold changes in KPNA2 mRNA and miR-26b expression were calculated using the $2^{-\Delta \Delta C t}$ method.

\section{Plasmids}

KPNA2 expression vector (pBabe-KPNA2) and OCT4 expression vector (pBabe-OCT4) were generated by respectively subcloning the full-length human KPNA2 and OCT4 cDNA into the pBABE-puro plasmid (Langri, GangZhou, China). pCDH-miR-26b were provided by Professor SM Zuang (Sun Yat-Sen University).

\section{Cell transfection}

The hsa-miR-26b mimics and scramble oligonucleotides were obtained from Ribobio (Ribobio, Guangzhou, China). Human KPNA2 siRNA (sc-35741) and OCT4 siRNA (sc-36123) was purchased from Santa Cruz Biotechnology (Santa Cruz, CA, USA). COV644 and OVCAR-3 cell lines were seeded on 6-well plates and transfected the next day using Lipofectamine 2000 (Invitrogen), according to the manufacturer's instructions. Cells were harvested $48 \mathrm{~h}$ after transfection for qRT-PCR analysis and western blotting.

\section{Western blot analysis}

Total cellular proteins were extracted and separated on SDS-PAGE gels, and western blotting was performed in accordance with standard procedures (for details, please see reference 4). GAPDH was used as a loading control on the same membrane. The primary antibodies used include anti-KPNA2 (ab82313, Abcam) (sc-6917, Santa Cruz), anti-OCT4 (ab19857, Abcam), anti-E-cadherin (610181, BD), anti-Vimentin (5741, Cell Signaling Technology) and anti-GAPDH (sc-32233, Santa Cruz).

\section{Luciferase assay}

A fragment of the 3'-UTR of KPNA2 mRNA (region 1723-2011, from NM_002266) and a mutant variant were cloned into the $X b \bar{a}$ I site of a pGL3-control vector (Promega); the new vectors were named pGL3KPNA2-WT and pGL3-KPNA2-MUT, respectively. $293 \mathrm{~T}$ and OVCAR-3 cells were seeded at $5 \times 10^{4}$ cells per well in a 24-well plate and co-transfected $24 \mathrm{~h}$ later with pGL3-control (200 ng), pGL3-KPNA2-WT (200 ng), pGL3-KPNA2-MUT (200 ng), pGL4.73 vector (10 ng; Promega), miR-26b mimics $(50 \mathrm{pmol})$ or scramble miRNA (50 pmol) using Lipofectamine 2000 (Invitrogen). Cells were harvested $48 \mathrm{~h}$ after transfection and luciferase activities were analyzed using the DualLuciferase Reporter Assay System in accordance with the manufacturer's instructions (Promega). Firefly luciferase activity was then normalized to that of Renilla luciferase. All experiments were performed in triplicate.

\section{In vitro proliferation assay}

An 3-(4,5-dimethylthiazole-2-yl)-2,5-biphenyl tetrazolium bromide (MTT) assay was used to detect cell proliferation. Briefly, cells (1500 cells/well) were seeded in 96-well plates. After transfection, the cells were cultured for the indicated time intervals and stained with sterile MTT dye $(0.5 \mathrm{mg} / \mathrm{ml}$, Sigma). After $4 \mathrm{~h}$ incubation, the supernatant was aspirated, and dimethyl sulfoxide (Sigma) was added. The absorbance was measured at 490 $\mathrm{nm}$. All experiments were performed in triplicate.

\section{In vitro migration assay}

Cell migratory potential was evaluated using transwell chambers ( $8 \mu \mathrm{m}$ pore; BD Biosciences). A cell suspension $\left(5 \times 10^{4}\right)$ in DMEM medium without serum was pipetted into the upper insert of a 24 -well chamber 24 $\mathrm{h}$ after transfection; the bottom chamber was filled with DMEM containing $10 \%$ FBS. After $24 \mathrm{~h}$, cells remaining on the upper surface of the membrane were removed. Cells that had migrated to the lower surface of the membrane were stained with crystal violet and five independent fields were counted under a microscope. All experiments were performed in triplicate.

\section{Sphere formation assay}

Per the method described by Dontu et al. [52] with minor modifiations, Single-cell suspensions were seeded at $5 \times 10^{3}$ cells per well in ultra-low attachment 6-well plates (Corning, Lowell, MA, USA), and cultured with DMEM/ F12 supplemented with $20 \mathrm{ng} / \mathrm{mL}$ epidermal growth factor (R\&D Systems), $20 \mathrm{ng} / \mathrm{mL}$ basic fibroblast growth factor (R\&D Systems), and B-27supplement (Gibco). The number and size of spheres formed were evaluated under microscopy after 10 days. All experiments were performed in triplicate. 


\section{In vivo proliferation and metastasis assay}

Female BABL/c nude mice (4-5 weeks old) were purchased from the Experimental Animal Center of Guangdong Province (Guangzhou, China). All animal studies were conducted in accordance with the National Institutes of Health's animal use guidelines and current national regulations and standards regarding the use of laboratory animals. All animal procedures were approved by the Sun Yat-sen University Institutional Animal Care and Use Committee. For tumor growth assays, OVCAR-3 cells stably overexpressing miR-26b or scramble miRNA were resuspended in PBS, and $5 \times 10^{5}$ cells $(200 \mu \mathrm{l})$ were subcutaneously injected into the dorsal flank of nude mice. Tumor size was measured every 3 days and tumor volumes were calculated with following formula: volume $=\left(\mathrm{L} \times \mathrm{S}^{2}\right) / 2$, in which $\mathrm{L}$ refers to the longest diameter and $\mathrm{S}$ shortest. Mice were sacrificed post three weeks, and tumors were dissected and weighted.

To investigate the effect of miR-26b on metastasis, OVCAR-3 cells stably overexpressing miR-26b or scramble miRNA $\left(1 \times 10^{6}\right.$ cells/mouse) were injected into the tail vein of nude mouse (six for each cell group). Six weeks after injection, the animals were sacrificed, and their lungs were dissected and paraffin-embedded. Consecutive sections $(5 \mu \mathrm{m})$ were stained with hematoxylin and eosin. The micrometastases in the lungs were counted under a dissecting microscope. To detect the expression of KPNA2 and OCT4 in vivo, we carried out immunohistochemistry analysis according to standard procedures.

\section{Statistical analysis}

Statistical analysis was performed using the SPSS software package (version 13.0, SPSS). Data is presented as the means $\pm \mathrm{SD}$ and was assessed using a two-tailed Student's $t$-test. A threshold for statistically significant differences was set at $P<0.05$. Regression analysis (Pearson's correlation) was used to assess the correlation of miR-26b and KPNA2 expression.

\section{ACKNOWLEDGMENTS}

This research was supported by grants from the National Natural Science Foundation of China (Grant numbers 81171948, 81372275), the Key Program of Natural Science Foundation of Guangdong Province, China (Grant number S2012020011060), the Project of State Key Laboratory of Oncology in South China (Grant number 030041060004) and the Project of Guangzhou science and technology plan (2014J4100238).

We thank Prof. Weng-Lin Huang, Prof. Li-Min Zheng and Dr. Jiang-Xue Wu for their kindly provision of experimental sites, technical guidance and paper revisions.

\section{CONFLICTS OF INTEREST}

The authors declare that there are no conflicts of interest.

\section{REFERENCES}

1. Siegel R, Ma J, Zou Z, Jemal A. Cancer statistics, 2014. CA Cancer J Clin. 2014; 64: 9-29.

2. Zheng Min, Tang Li, Huang Long, Ding Hui, Liao WenTing, Zeng Mu-Sheng, Wang Hui-Yun. Overexpression of karyopherin-2 in epithelial ovarian cancer and correlation with poor prognosis. Obstet Gynecol. 2010; 116: 884-891.

3. Li He, Hui Ding, Jian-Hua Wang, Yun Zhou, Li Li, YanHong Yu, Long Huang, Wei-Hua Jia, Musheng Zeng, JingPing Yun, Rong-Zhen Luo, Min Zheng. Overexpression of karyopherin 2 in human ovarian malignant germ cell tumor correlates with poor prognosis. PLoS One. 2012;7: e42992.

4. L Huang, H-Y Wang, J-D Li, J-H Wang, Y Zhou, R-Z Luo, J-P Yun, Y Zhang, W-H Jia and M Zheng. KPNA2 promotes cell proliferation and tumorigenicity in epithelial ovarian carcinoma through upregulation of c-Myc and downregulation of FOXO3a. Cell Death Dis. 2013;4: e745.

5. Tseng SF, Chang CY, Wu KJ, Teng SC. Importin KPNA2 is required for proper nuclear localization and multiple functions of NBS1. J Biol Chem. 2005; 280: 39594-39600.

6. Laura Zannini, Daniele Lecis, Sofia Lisanti, Roberta Benetti, Giacomo Buscemi,Claudio Schneider, Domenico Delia. Karyopherin-alpha2 protein interacts with Chk2 and contributes to its nuclear import. J Biol Chem. 2003; 278: 42346-42351.

7. Teng SC, Wu KJ, Tseng SF, Wong CW, Kao L. Importin KPNA2, NBS1, DNA repair and tumorigenesis. J Mol Histol. 2006; 37: 293-299.

8. Edgar Dahl, Glen Kristiansen, Kathrin Gottlob, Irina Klaman, Elke Ebner, Bernd Hinzmann, Klaus Hermann, Christian Pilarsky, Matthias Durst, Monika KlinkhammerSchalke, Hagen Blaszyk, Ruth Knuechel, Arndt Hartmann, et al. Molecular profiling of laser-microdissected matched tumor and normal breast tissue identifies karyopherin alpha2 as a potential novel prognostic marker in breast cancer. Clin Cancer Res. 2006; 12: 3950-3960.

9. Christos Sotiriou, Pratyaksha Wirapati, Sherene Loi, Adrian Harris, Steve Fox, Johanna Smeds, Hans Nordgren, Pierre Farmer, Viviane Praz, Benjamin Haibe-Kains, Christine Desmedt, Denis Larsimont, Fatima Cardoso, et al. Gene expression profiling in breast cancer: understanding the molecular basis of histologic grade to improve prognosis. J Natl Cancer Inst. 2006; 98: 262-272.

10. Anja Dankof, Florian R. Fritzsche, Edgar Dahl, Stefan Pahl, Peter Wild, Manfred Dietel, Arndt Hartmann, Glen Kristiansen. KPNA2 protein expression in invasive breast carcinoma and matched peritumoral ductal carcinoma in situ. Virchows Arch. 2007; 451: 877-881. 
11. Oleg Gluz, Peter Wild, Robert Meiler, Raihana DialloDanebrock, Evelyn Ting, Svjetlana Mohrmann, Gerhart Schuett, Edgar Dahl, Thomas Fuchs, Alexander Herr, Andreas Gaumann, Markus Frick, Christopher Poremba, et al. Nuclear karyopherin alpha2 expression predicts poor survival in patients with advanced breast cancer irrespective of treatment intensity. Int J Cancer. 2008; 123: 1433-1438.

12. Makoto Sakai, Makoto Sohda, Tatsuya Miyazaki, Shigemasa Suzuki, Akihiko Sano, Naritaka Tanaka, Takanori Inose, Masanobu Nakajima, Hiroyuki Kuwano. Significance of karyopherin-\{alpha\} 2 (KPNA2) expression in esophageal squamous cell carcinoma. Anticancer Res. 2010; 30: 851-856.

13. Chun-I. Wang, Chih-Liang Wang, Chih-Wei Wang, ChiDe Chen, Chih-Ching Wu, Ying Liang, Ying-Huang Tsai, Yu-Sun Chang, Jau-Song Yu and Chia-Jung Yu. Importin subunit alpha-2 is identified as a potential biomarker for non-small cell lung cancer by integration of the cancer cell secretome and tissue transcriptome. Int J Cancer. 2011; 128: 2364-2372.

14. Ashkan Mortezavi, Thomas Hermanns, Hans-Helge Seifert, Martin K. Baumgartner, Maurizio Provenzano, Tullio Sulser, Maximilian Burger, Matteo Montani, Kristian Ikenberg, Ferdinand Hofstadter, Arndt Hartmann, Rolf Jaggi, Holger Moch, et al. KPNA2 expression is an independent adverse predictor of biochemical recurrence after radical prostatectomy. Clin Cancer Res. 2011; 17: 1111-1121.

15. Kenichiro Yoshitake, Shinji Tanaka, Kaoru Mogushi, Arihiro Aihara, Ayano Murakata, Satoshi Matsumura, Yusuke Mitsunori, Mahmut Yasen, Daisuke Ban, Norio Noguchi, Takumi Irie, Atsushi Kudo, Noriaki Nakamura, et al. Importin-alphal as a novel prognostic target for hepatocellular carcinoma. Ann Surg Oncol. 2011; 18: 2093 2103.

16. Jørgen Bjerggaard Jensen, Pia Pinholt Munksgaard, Christoffer Mørk Sørensen, Niels Fristrup, Karin Birkenkamp-Demtroder, Benedicte Parm Ulhøi, Klaus Møller-Ernst Jensen, Torben F. Ørntoft, Lars Dyrskjøt. High expression of karyopherin-alpha2 defines poor prognosis in non-muscle-invasive bladder cancer and in patients with invasive bladder cancer undergoing radical cystectomy. Eur Urol.2011; 59: 841-848.

17. Gousias K, Becker AJ, Simon M, Niehusmann P. Nuclear karyopherin a2: a novel biomarker for infiltrative astrocytomas. J Neurooncol. 2012; 109: 545-553.

18. Noetzel E, Rose M, Bornemann J, Gajewski M, Knuchel R, Dahl E. Nuclear transport receptor karyopherin-alpha2 promotes malignant breast cancer phenotypes in vitro. Oncogene. 2012; 31: 2101-2114.

19. Yasuhara N, Shibazaki N, Tanaka S, Nagai M, Kamikawa Y, Oe S, Asally M, Kamachi Y, Kondoh H, Yoneda Y. Triggering neural differentiation of ES cells by subtype switching of importin-alpha. Nat Cell Biol. 2007; 9:72-79.

20. Xiangqun Li, Lei Sun, Ying Jin. Identification of karyopherin-alpha 2 as an Oct4 associated protein. J Genet Genomics. 2008; 35:723-728.

21. Xiao-Lei Li, Lan-Ling Jia, Mu-Mu Shi, Xin Li, ZhongHua Li, Hui-Feng Li, En-Hua Wang, Xin-Shan Jia. Downregulation of KPNA2 in non-small-cell lung cancer is associated with Oct4 expression. J Transl Med. 2013; 11:232.

22. Scholer H, S Ruppert, N Suzuki, K Chowdhury, P Gruss. New type of POU domain in germ line-specific protein Oct4. Nature 1990; 344:435-9.

23. Guang Jin PAN, Zeng Yi CHANG, Hans R. SCHÖLER, Duanqing PEI. Stem cell pluripotency and transcription factor Oct4. Cell Res. 2002; 12: 321-329.

24. Schoenhals M, Kassambara A, De Vos J, Hose D, Moreaux J, Klein B. Embryonic stem cell markers expression in cancers. Biochem Biophys Res Commun. 2009; 383:157162.

25. Zeineddine D, Hammoud AA, Mortada M, Boeuf H. The Oct4 protein: more than a magic stemness marker. Am J Stem Cells. 2014; 3:74-82.

26. Bapat SA, Mali AM, Koppikar CB, Kurrey NK. Stem and progenitor-like cells contribute to the aggressive behavior of human epithelial ovarian cancer. Cancer Res. 2005; 65:3025-3029.

27. Peng S, Maihle NJ, Huang Y. Pluripotency factors Lin 28 and Oct4 identify a sub-population of stem cell-like cells in ovarian cancer. Oncogene. 2010; 29:2153-2159.

28. He L, Hannon GJ. MicroRNAs: small RNAs with a big role in gene regulation. Nat Rev Genet. 2004; 5: 522-531.

29. Ambros V. The functions of animal microRNAs. Nature. 2004; 431: 350-355.

30. Calin GA, Croce CM. MicroRNA signatures in human cancers. Nat Rev Cancer. 2006; 6: 857-866.

31. Bueno MJ, Perez de Castro I, Malumbres M. Control of cell proliferation pathways by microRNAs. Cell Cycle. 2008; 7: 3143-3148.

32. Nicoloso MS, Spizzo R, Shimizu M, Rossi S, Calin GA. MicroRNAs-the micro steering wheel of tumour metastases. Nat Rev Cancer. 2009; 9: 293-302.

33. Pablo Landgraf, Mirabela Rusu, Robert Sheridan, Alain Sewer, Nicola Iovino, Alexei Aravin, Sébastien Pfeffer, Amanda Rice, Alice O. Kamphorst, Markus Landthaler, Carolina Lin, Nicholas D. Socci, Leandro Hermida, et al. A mammalian microRNA expression atlas based on small RNA library sequencing. Cell. 2007; 129: 1401-1414.

34. Jun Lu, Gad Getz, Eric A. Miska, Ezequiel AlvarezSaavedra, Justin Lamb, David Peck, Alejandro SweetCordero, Benjamin L. Ebert, Raymond H. Mak, Adolfo A. Ferrando, James R. Downing, Tyler Jacks, H. Robert Horvitz, et al. MicroRNA expression profiles classify human cancers. Nature. 2005; 435: 834-838.

35. Stefano Volinia, George A. Calin, Chang-Gong Liu, Stefan Ambs, Amelia Cimmino, Fabio Petrocca, Rosa Visone, Marilena Iorio, Claudia Roldo, Manuela Ferracin, Robyn 
L. Prueitt, Nozumu Yanaihara, Giovanni Lanza, et al. A microRNA expression signature of human solid tumors defines cancer gene targets. Proc Natl Acad Sci U S A. 2006; 103: 2257-2261.

36. Bartel DP. MicroRNAs: genomics, biogenesis, mechanism, and function. Cell. 2004; 116: 281-297.

37. Ning Wu, Xiangzhong Zhao, Ming Liu, Haizhou Liu, Weicheng Yao, Yuyan Zhang, Shousong Cao, Xiukun Lin. Role of microRNA-26b in glioma development and its mediated regulation on EphA2. PLoS One. 2011; Vol. 6, pp. e16264.

38. Xiao-Xiao Liua, Xiao-Jun Li, Bo Zhang, Yong-Jun Liang, Ci-Xiang Zhou, Dan-Xia Cao, Ming He, GuoQiang Chen, Jian-Rong He, Qian Zhao. MicroRNA-26b is underexpressed in human breast cancer and induces cell apoptosis by targeting SLC7A11. FEBS Lett. 2011; 585: 1363-1367.

39. Li J, Kong X, Zhang J, Luo Q, Li X, Fang L. MiRNA-26b inhibits proliferation by targeting PTGS2 in breast cancer. Cancer Cell Int. 2013; 13: 7.

40. Zhang C, Tong J, Huang G. Nicotinamide phosphoribosyl transferase (Nampt) is a target of microRNA-26b in colorectal cancer cells. PLoS One. 2013;8: e69963.

41. Zhao N, Wang R, Zhou L, Zhu Y, Gong J, Zhuang SM. MicroRNA-26b suppresses the NF-kappaB signaling and enhances the chemosensitivity of hepatocellular carcinoma cells by targeting TAK1 and TAB3. Mol Cancer. 2014;13: 35.

42. Ying Zhu, Yang Lu, Qi Zhang, Jing-Jing Liu, Tuan-Jie Li, Jian-Rong Yang, Chunxian Zeng and Shi-Mei Zhuang. MicroRNA-26a/b and their host genes cooperate to inhibit the G1/S transition by activating the $\mathrm{pRb}$ protein. Nucleic Acids Res. 2012; 40: 4615-4625.

43. Holger Dill, Bastian Linder, Alexander Fehr, Utz Fischer. Intronic miR-26b controls neuronal differentiation by repressing its host transcript, ctdsp2. Genes Dev. 2012; 26:25-30.

44. Hans-Ingo Trompeter, Janine Dreesen, Eugenie Hermann, Katharina M Iwaniuk, Markus Hafner, Neil Renwick, Thomas Tuschl and Peter Wernet. MicroRNAs miR-26a, miR-26b, and miR-29b accelerate osteogenic differentiation of unrestricted somatic stem cells from human cord blood. BMC Genomics. 2013; 14:111.

45. Yan-Lei Ma, Peng Zhang, Feng Wang, Mary Pat Moyer, Jian-Jun Yang, Zhi-Hua Liu, Jia-Yuan Peng, Hong-Qi Chen, Yu-Kun Zhou, Wei-Jie Liu, Huan-Long Qin. Human embryonic stem cells and metastatic colorectal cancer cells shared the common endogenous human microRNA-26b. J Cell Mol Med. 2011; 15:1941-54.

46. Lin Zhang, Stefano Volinia, Tomas Bonome, George Adrian Calin, Joel Greshock, Nuo Yang, Chang-Gong Liu, Antonis Giannakakis, Pangiotis Alexiou, Kosei Hasegawa, Cameron N. Johnstone, Molly S. Megraw, Sarah Adams, et al. Genomic and epigenetic alterations deregulate
microRNA expression in human epithelial ovarian cancer. Proc Natl Acad Sci U S A. 2008; 105: 7004-7009.

47. Eun Ji Nam, Heejei Yoon, Sang Wun Kim, Hoguen Kim, Young Tae Kim, Jae Hoon Kim, Jae Wook Kim, and Sunghoon Kim. MicroRNA expression profiles in serous ovarian carcinoma. Clin Cancer Res. 2008; 14: 2690-2695.

48. Ye-Won Chung, Hyo-Sook Bae, Jae-Yun Song, Jae Kwan Lee, Nak Woo Lee, Tak Kim, and Kyu-wan Lee. Detection of microRNA as novel biomarkers of epithelial ovarian cancer from the serum of ovarian cancer patients. Int $\mathrm{J}$ Gynecol Cancer. 2013; 23: 673-679.

49. Kamikawa Y, Yasuhara N, Yoneda Y. Cell type-specific transcriptional regulation of the gene encoding importinalpha1. Exp Cell Res. 2011; 317: 1970-1978.

50. van der Watt PJ, Ngarande E, Leaner VD. Overexpression of Kpnbeta1 and Kpnalpha2 importin proteins in cancer derives from deregulated E2F activity. PLoS One. 2011;6: e27723.

51. Chun-I Wang, Kun-Yi Chien, Chih-Liang Wang, Hao-Ping Liu, Chia-Chen Cheng, Yu-Sun Chang, Jau-Song Yu, and Chia-Jung Yu. Quantitative proteomics reveals regulation of karyopherin subunit alpha-2 (KPNA2) and its potential novel cargo proteins in nonsmall cell lung cancer. Mol Cell Proteomics. 2012; 11: 1105-1122.

52. Gabriela Dontu, Wissam M. Abdallah, Jessica M. Foley, Kyle W. Jackson, Michael F. Clarke, Mari J. Kawamura, and Max S. Wicha. In vitro propagation and transcriptional profiling of human mammarystem/progenitor cells. Genes Dev. 2003;17: 1253-1270. 